\title{
Visualisation of compliant declarative business processes
}

\author{
Nina Ghanbari Ghooshchi ${ }^{1}$, Nick van Beest ${ }^{2}$, Guido Governatori ${ }^{2}$, Francesco Olivieri ${ }^{2}$, Abdul Sattar ${ }^{1}$ \\ ${ }^{1}$ Griffith University, Australia \\ nina.ghanbari@griffithuni.edu.au, a.sattar@griffith.edu.au \\ ${ }^{2}$ Data61, CSIRO, Spring Hill, Australia \\ \{nick.vanbeest, guido.governatori, francesco.olivieri\}@data61.csiro.au
}

\begin{abstract}
Organisations typically have to cope with large numbers of business rules and existing regulations governing the business in which they operate. Due to the size and complexity of those rules, maintenance is difficult and it is increasingly complicated to ensure that each business process adheres to those rules. As such, automated extraction of business processes from rules has a number of clear advantages: (1) visualisation of all possible executions allowed by the rules, (2) automated execution and compliance by design, (3) identification of "inefficiencies" in the business rules. Existing approaches, however, only allow to generate partial traces based on input specifications and cannot handle many different input cases resulting in a full process. This paper presents a formal method to visualise and operationalise such sets of rules as a verifiable business process that is compliant by design and allows us to analyse all possible execution paths. In addition, it maintains information of all distinct input cases, to preserve dependencies between consecutive exclusive paths.
\end{abstract}

\section{INTRODUCTION}

It is a common scenario that organisations develop business rules manuals for their operations. Such business rules may specify constraints that apply to their business processes (e.g. a customer has to be older than 18 in order to be eligible for a loan). As organisations grow, so do their processes and business rules. As a consequence, the number of business rules is generally large [1].

In addition, organisations have to comply with regulations governing the business in which they operate. The compliance rules resulting from these regulations affect the procedures and processes described by the business rules. Although the combination of business rules and compliance rules allows organisations to determine whether such procedures and processes are compliant with the regulatory requirements, in general, for large rule sets the relationships among the rules (and the activities and conditions they describe) are difficult to understand and maintenance of those rule set is complicated [1]. Furthermore, it is difficult to ensure that each business process adheres to those rules [2].

However, the combination of these rules implicitly contains the processes for organisations, as the constraints are specified by those rules. The extraction of business processes from rules has a number of clear advantages: (1) it helps to visualise all possible executions allowed by the rules, (2) the rules can be implemented by a workflow engine for automated execution and compliance by design, (3) it helps to identify "inefficiencies" in the business rules (e.g. rules with side effects that do not contribute to the goals of a process, multiple rules for the same goal, etc).

We adopt, as a starting point to create our business processes, a rule-based formalism because rules are well suited to describe both the external constraints regulating the setting (norms), and how the agent perceives the world (beliefs), i.e., which actions the agent may undertake. In this way, rules are able to define the relationships between states of the world.

Indeed, rules are able to define relationships among the tasks to be executed to achieve a goal, and are useful in understanding the behaviour of such tasks. By shifting the focus from the entire process to its basic building blocks, it can be identified which preconditions trigger the activation of a task, as well as what happens once a task completes its execution. This way of describing processes provides knowledge concerning the reasons why to execute a certain task instead of another and, implicitly, defines the control flow of a process describing the situations that do not violate any of the given relationships.

Existing approaches, however, only allow to generate partial traces based on input specifications [3], [4], and cannot handle many different input cases resulting in a full process [5] or duplicate transitions to represent every possible trace [6]. Therefore, to tackle this problem, this paper presents a formal method to visualise and operationalise such sets of rules as a verifiable business process (i.e., it is possible to check whether the process is sound [7] and compliant [8]). The generated business process is compliant by design and allows us to analyse all possible execution paths within the rules. In addition, the generated business process is optimised for execution of the business rules and regulations, as it is specifically designed to exactly represent the behaviour allowed by the rules.

The remainder of this paper is structured as follows. In Section II, we discuss the background with respect to rules for declarative process modelling. Subsequently, Section III describes the preliminary definitions related to Petri nets. The method and the corresponding algorithms are described in Section IV, along with running examples to illustrate the core idea. Lastly, Section V presents the related work, while Section VI concludes the paper and discusses future work.

\section{Rules for Declarative Processes}

Governatori et al. [9], [10] proposed an agent-oriented rule language for the declarative specifications of norm and goal compliant business processes. The main idea is that the set of rules can be partitioned into three subsets: a set of rules corresponding to the norms governing a process (obligation rules), a set of rules encoding the objectives/goals of an organisation to fulfil in their processes (outcome rules) and, finally, a set of rules describing the "capabilities" of an organisation. The intuition behind the capability rules is that they model the set of activities/tasks an organisation is able to carry out, the preconditions required for each task, the effects 
of such tasks, and the relationships among tasks. The language upon which the rules are defined consists of a set of two types of literals: condition literals and task literals. The condition literals encode the preconditions and effects of tasks or, in general, state variables for a process, while each task literal corresponds to a task that could occur in a process.

Capability rules have an "if... then..." form: $r: l_{1}, \ldots, l_{n} \Rightarrow l_{n+1}$, where $r$ is a label that uniquely identifies the rule, and each literal $l_{i}$ is drawn from the set of literals (atomic propositions and their negation) representing conditions $c_{i}$ and tasks $t_{j}$ (a positive task literal means that the task is executed in a process, while the negation, i.e., $\neg t_{k}$ means that task $t_{k}$ does not appear in the process). This form has the clear advantage that it immediately relates preconditions to the corresponding effect of performing the particular action. More specifically, we can identify the following three patterns. (1) $t \Rightarrow c$, where we can look at $c$ as an effect of performing task $t$; thus the effect represented by $c$ holds after the execution of task $t$. (2) The pattern $c_{1}, \ldots c_{n} \Rightarrow t$ indicates that $c_{1}, \ldots c_{n}$ are preconditions for tasks $t$, and task $t$ will be executed after the preconditions hold. (3) $t_{1}, \ldots, t_{n} \Rightarrow t$ specifies that the combination of tasks $t_{1}, \ldots, t_{n}$ triggers task $t$, and that task $t$ appears in the process, if $t_{1}, \ldots, t_{n}$ appear in the process (before $t$ ). In other words, this pattern describes relationships and dependencies among tasks in a process. In the rule given above, the meaning is that execution of tasks $t_{1}, \ldots, t_{n}$ is required to trigger the execution of task $t$.

The rules are then used to form (logical) derivations, where a derivation $D$, given a set of facts $F$ represented as literals, is a sequence of literals $D(1), \ldots, D(n)$, such that if $D(m+1)=l$ then either $l \in F$ or there is a rule $r: l_{1}, \ldots, l_{k} \Rightarrow l$ such that for all $l_{i} \in D[1 . . m], i \leq k$, where $D[1 . . m]$ is the initial sequence of length $m$ of $D$.

The rules presented above can be linked to the sequential, parallel, and alternative patterns typical of business process modelling techniques to those that can be found in a logical derivation. For instance, assume tasks $A$ and $B$ concur to obtain the resources needed for task $C$ to start its execution. This means that $C$ may bring about its effects only when both $A$ and $B$ have finished, and that $A$ and $B$ have no precedence order with respect to one another, that is they can be executed in parallel. From a logical perspective, all this information can simply be represented by a rule with premises $A$ and $B$ and a conclusion $C$, i.e., something of the form ' $A, B \Rightarrow C$ '. Accordingly, a derivation can encode a possible order in which the tasks are executed to achieve a particular business goal according to the constraints specified by the rules themselves.

Given a set of facts, we can generate a derivation where all applicable rules fire and their conclusions have been added to the derivation. This derivation contains all tasks that are executable/executed, given the set of facts that can be considered as the input for a process case (and instance of a business process). In addition, the derivation contains the literals corresponding to the conditions to trigger the execution of tasks or for activating obligations, the effects of the tasks, the obligations in force, and the expected goals. While obligations and goals determine whether an execution of the process is compliant and meet the organisation objectives (thereby influencing the activities or tasks included in the process), they are not actions nor tasks. Therefore, rules for goals and norms do not directly contribute to the structure of the process: they only provide constraints on the tasks that can appear in a derivation. Goals and obligations can thus be considered as special kinds of conditions. Accordingly, if one ignores obligations, goals and the condition literals from a derivation, then a derivation is a sequence of tasks satisfying the constraints defined by the rules. This means that it is equivalent to a plan as defined in classical planning [11]. For these reasons, we concentrate only on the capability rules in the remainder of this paper.

Notice that, while the set of tasks triggered by a case (set of facts) is unique, multiple derivations are possible. For example, given the rule $t_{1}, t_{2} \Rightarrow t_{3}$, the order in which $t_{1}$ and $t_{2}$ are executed does not matter. Accordingly, both $t_{1}, t_{2}, t_{3}$ and $t_{2}, t_{1}, t_{3}$ are valid derivations (and, consequently, plans conforming to the specification given by the rule). This means that, given a case, we can generate a set of plans corresponding to it, which can be understood as alternative ways in which the process can be executed. Using the idea that a business process can be understood by a set of traces (where a trace is a sequence of tasks), we can establish a connection between a set of plans and a business process, where a process provides a concise (formal and graphical) representation of a set of plans, which are obtained from a single case and are combined using constructions modelling and-splits and and-joins. Moreover, given a set of rules, it is possible to give as input mutually exclusive sets of facts, where, for instance, each set of facts corresponds to a common set of instances for the process. For each case, a corresponding set of plans is created, where the mutually exclusive cases are subsequently merged, adopting xor-splits and xor-joins.

\section{Petri nets}

Business processes can be modelled by using many different notations. Often these notations require further formalisation before formal verification can be applied. Petri nets (PN) [12] are a popular modelling language used to formalise business processes [7]. Petri nets are directed bi-graphs with nodes consisting of places and transitions, representing conditions and events respectively. Arcs form directed edges between placetransition pairs. Places may contain tokens. A distribution of tokens over the places is called a marking. A transition is enabled and can "fire" when all its input places contain at least one token. When a transition fires, one token is removed from each input place and one token is put into each output place. A labelled Petri net [12] is defined formally as a tuple $(P, T, A, \lambda)$ where $P$ is a set of places, $T$ is a set of transitions, such that $P \cap T=\emptyset, A \subseteq(P \times T) \cup(T \times P)$ is a set of arcs and $\lambda: P \cup T \rightarrow \mathcal{L}$ is a labelling function. The Petri net state, often referred to as the net marking, $M: P \rightarrow \mathbb{N}_{0}$ is a function that associates a place $p \in P$ with a natural number (viz., place tokens). A marked net $N=\left(P, T, A, \lambda, M_{0}\right)$ is a Petri net $(P, T, A, \lambda)$ together with an initial marking $M_{0}$.

The preset of a node is denoted by $\bullet y=\{x \in P \cup T \mid(x, y) \in$ $A\}$, and the postset of a node is denoted by $y \bullet=\{z \in P \cup$ $T \mid(y, z) \in A\}$. If $\forall p \in \bullet t: M(p)>0, t$ is said to be enabled. The firing of $t$, denoted by $M \longrightarrow M^{\prime}$, leads to a new marking $M^{\prime}$, with $M^{\prime}(p)=M(p)-1$ if $p \in \bullet \backslash t \bullet, M^{\prime}(p)=M(p)+1$ if $p \in t \bullet \backslash \bullet t$, and $M^{\prime}(p)=M(p)$ otherwise. The marking $M_{n}$ is said to be reachable from $M$ if there exists a sequence 
of transition firings $\sigma=t_{1} t_{2} \ldots t_{n}$ such that $M \stackrel{t_{1}}{\longrightarrow} M_{1} \stackrel{t_{2}}{\longrightarrow}$ $\ldots \stackrel{t_{n}}{\longrightarrow} M_{n}$.

A trace is a sequence $\lambda\left(t_{1}\right), \lambda\left(t_{2}\right), \ldots$ such that $\sigma=t_{1}, t_{2}, \ldots$ is a sequence of firing transitions. However, certain controlflow behaviour (like exclusive parallel branches) requires additional transitions that do not correspond to a task literal. These transitions are commonly referred to as silent or $\tau$ transitions [13]. For understandability purposes, we will add a label for each $\tau$ transition as well throughout the paper. As such, the set of transition labels $\mathcal{L}$ comprises both labels corresponding to task literals and labels corresponding to $\tau$ transitions. A visible trace is a trace where all $\tau$ transitions have been removed (maintaining the order of the transitions representing task literals). For the remainder of this work, we shall refer to visible traces as traces.

\section{From Rules to Petri Nets}

\section{A. Method}

In order to transform a set of rules $R$ representing the norms of an organisation to a Petri net $N$, a number of steps are required, which are shown graphically in Figure 1.

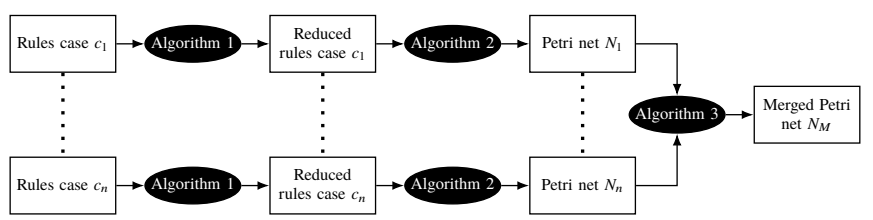

Fig. 1: Method overview.

First, pre-processing of the rules is required to take into account only those rules that define the possible executions in the process. Given that in this phase we already know where the execution is compliant, obligations and outcomes are transformed into conditions. From the resulting set of capability rules, we remove all rules with literals that have not been proved, as these rules cannot fire and as such have no effect on the resulting process.

The resulting set of (pre-processed) rules will be used to produce the Petri net. However, only tasks that are potentially executed should be represented in $N$. As negative tasks represent the absence of a task in the execution, these can be eliminated from the antecedents of the rules (e.g. $A, \neg B \Rightarrow C$ would be $A \Rightarrow C$ ). These negative tasks can be treated as additional conditions, which are satisfied automatically as they do not appear in the resulting net $N$ and can, therefore, not be executed. In addition, rules containing a negative task in the consequence (e.g. $A \Rightarrow \neg B$ ) can be removed, as these rules result in the absence of a task, which is automatically satisfied by not including the respective task in the resulting net $N$. The removal of negative tasks is shown in Algorithm 1 below.

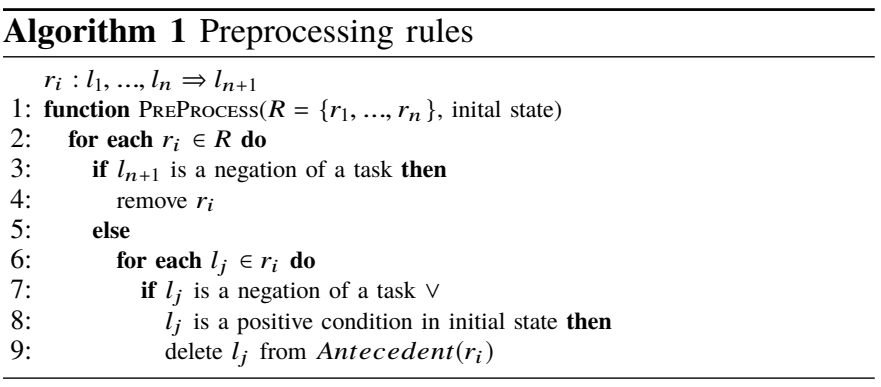

\section{B. Obtaining a Petri net from rules}

Once we obtained the reduced rule set for each case $c_{i}$, we can subsequently transform each set of reduced rules to a Petri net $N_{i}$ corresponding to that case by using Algorithm 2. That is, the traces of each $N_{i}$ contain exactly all possible derivations from the rules of its corresponding case $c_{i}$.

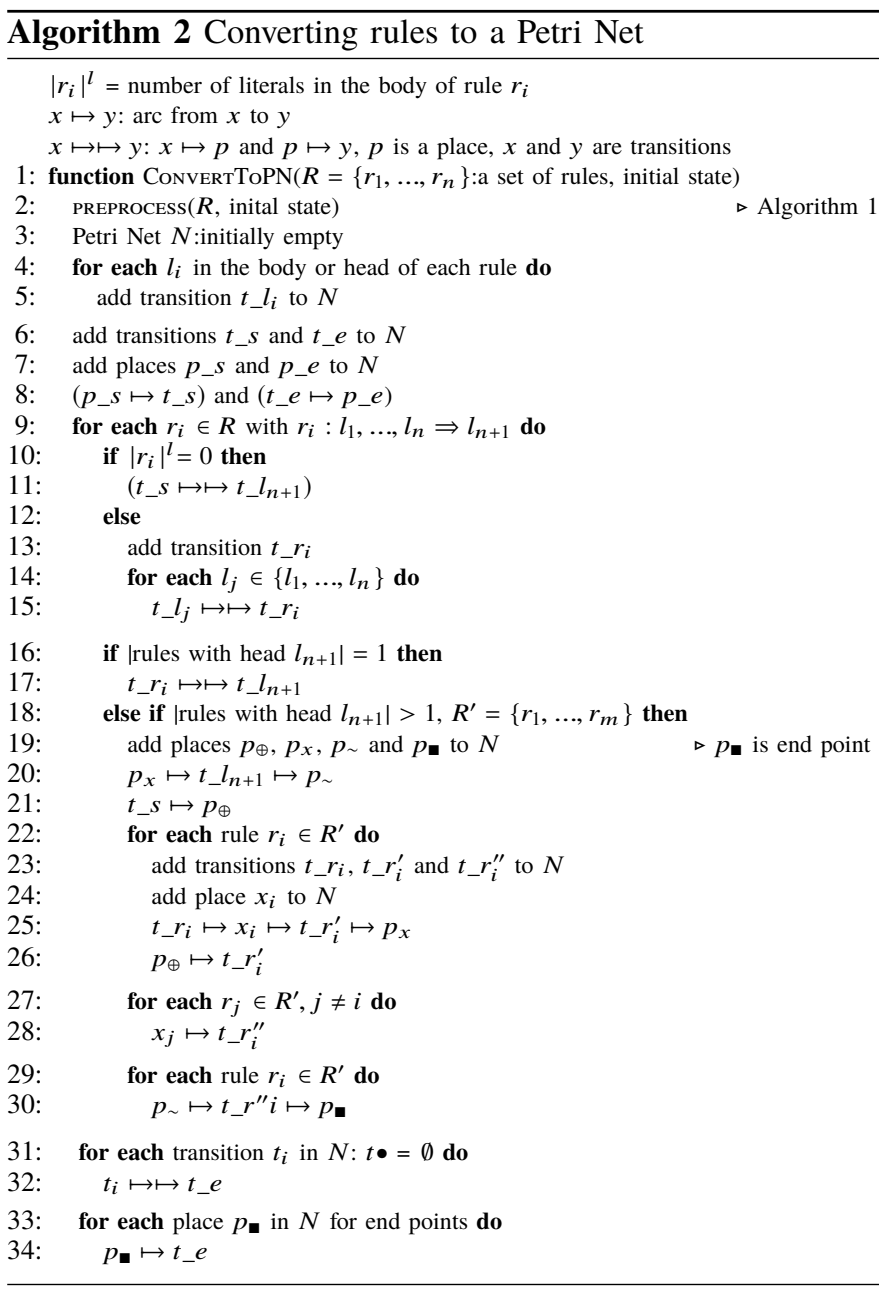

The net $N_{i}$ starts with an initial place $p_{s}$ and subsequent transition $t_{s}$.

$$
\text { (p) } \rightarrow t_{s}
$$

From there, the initial rules (i.e. rules with empty antecedent) are added. For example, a rule $r_{0}: \Rightarrow A$ would add an additional place and a transition $A$ after $t_{s}$.

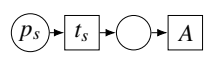

A rule $r_{1}: A \Rightarrow B$ first adds a $\tau$ transition representing $r_{1}$, followed by a place and a transition $B$ after $A$, to realise the sequence from $A$ to $B$ induced by $r_{1}$.

$$
\cdots \rightarrow A \rightarrow \bigcirc \rightarrow \tau \rightarrow \bigcirc \rightarrow B
$$

Similarly, adding a rule $r_{2}: A \Rightarrow C$ would add another outgoing place from $\mathrm{A}$ followed by a similar construct as created by $r_{1}$ leading to a transition $C$. A rule with multiple tasks in its antecedent, e.g. $r_{3}: B, C \Rightarrow D$, results in an ANDjoin of $B$ and $C$ to $D$. 


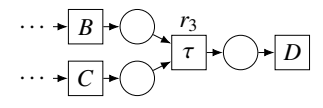

Finally, the last transitions are joined into a common end transition $t_{e}$, which leads to the sink place $p_{e}$.

$$
\cdots \rightarrow \bigcirc-t_{e} \rightarrow P_{e}
$$

When merged together, the resulting net can be visualised as follows:

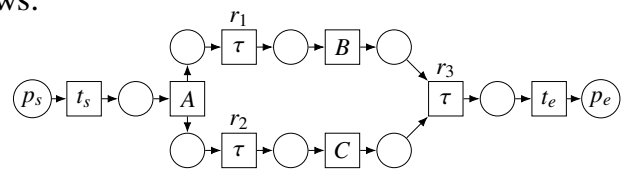

These basic principles work well for simple scenario's that result in a structured Petri net (i.e. every split has its matching join). However, when two concurrent paths lead into the same transition without synchronisation, this may cause this transition to be executed more than once.

This can easily be illustrated by replacing $r_{3}$ in the previous example with $r_{4}: B \Rightarrow D$ and $r_{5}: C \Rightarrow D$. It is straightforward to see that the execution of $A$ activates both branches,

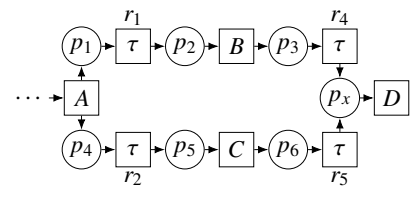
resulting in two tokens in $p_{x}$. Therefore, an additional construct is required to allow either $r_{4}$ or $r_{5}$ to produce a token for $p_{x}$ and remove the remaining tokens. As such, we require two auxiliary transitions $r_{4}^{\prime}$ (producing a token for $p_{x}$ ) and $r_{4}^{\prime \prime}$ (cleaning up remaining tokens). Similar for $r_{5}$. When $r_{4}^{\prime}$ fires, $r_{4}^{\prime \prime}$ cannot and vice versa, whereas when $r_{5}^{\prime}$ fires, $r_{5}^{\prime \prime}$ cannot and vice versa. A common input place $p_{\oplus}$ is introduced for $r_{4}^{\prime}$ and $r_{5}^{\prime}$, to allow only one of them to fire. As a result, firing of $r_{4}^{\prime}$ coincides with $r_{5}^{\prime \prime}$ and firing of $r_{5}^{\prime}$ coincides with $r_{4}^{\prime \prime}$.

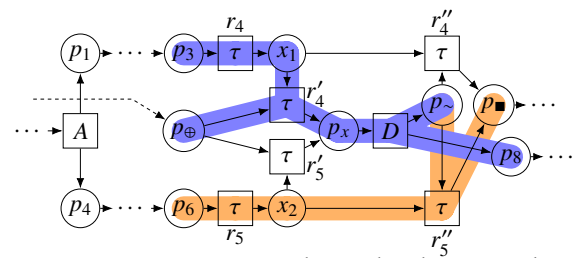

Let us assume a scenario where both $r_{4}$ and $r_{5}$ have fired, resulting in a token in $x_{1}$ and a token in $x_{2}$ respectively. If $r_{4}^{\prime}$ fires, it consumes tokens from $p_{\oplus}$ and $x_{1}$ and produces a token to $p_{x}$ to allow $D$ to fire and produce a token to $p_{8}$ and auxiliary place $p_{\sim}$ (highlighted in purple). The remaining token in $x_{2}$ is consumed along with the token in $p_{\sim}$ by $r_{5}^{\prime \prime}$, which produces a token for $p_{\text {. }}$ (highlighted in orange). Place $p_{8}$ is then connected to any subsequent rules having $D$ in their antecedent (e.g. $r_{6}: D \Rightarrow E$ ), whereas $p_{\mathbf{m}}$ is connected to the final transition to ensure that all remaining tokens are consumed. This construct ensures that, while having two rules producing $D$, only one execution of $D$ is possible, without having any orphaned tokens after finishing execution of the process. The entire net can be visualised as shown in Figure 2.

\section{Merging cases}

The algorithm described in the previous subsection is used for transforming individual cases into Petri nets. The final step is to merge the Petri nets of these individual cases into a combined Petri net that represents the entire process, which is achieved through Algorithm 3.

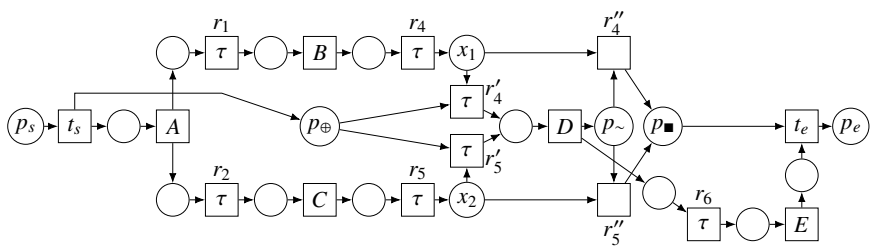

Fig. 2: Petri net showing the merge of two branches without synchronisation.

Let us illustrate the merging with a small example, where we have two different cases: Case $c_{1}=\left\{r_{1}: \Rightarrow A, r_{2}: A \Rightarrow C, r_{3}\right.$ : $C \Rightarrow D\}$ and Case $c_{2}=\left\{r_{4}: \Rightarrow B, r_{5}: B \Rightarrow C, r_{6}: C \Rightarrow E\right\}$. The Petri nets $N_{1}$ and $N_{2}$, representing $c_{1}$ and $c_{2}$ respectively, are shown graphically in Figure 3. Note that the two cases differ on the first and final task to be executed, but contain a mutual task $C$ in between.

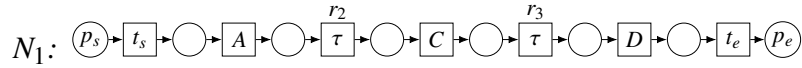

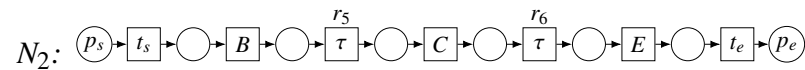

Fig. 3: Petri nets $N_{1}$ and $N_{2}$ for cases $c_{1}$ and $c_{2}$.

Furthermore, $A$ co-occurs with $D$ and $B$ co-occurs with $E$, but $A$ and $E$ or $B$ and $D$ cannot occur in the same trace. As such, $p_{s}$ and $t_{s}$ are common,

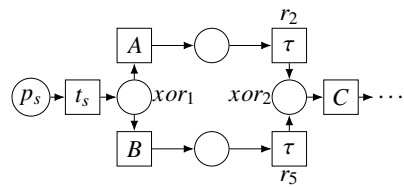
where the outgoing place (labeled $x_{0} r_{1}$ ) of $t_{s}$ marks the split to either $A$ (case $c_{1}$ ) or $B$ (case $c_{2}$ ). Next, $A$ leads to $C$ via $r_{2}$, whereas $B$ leads to $C$ via $r_{5}$, having $x_{0} r_{2}$ as xor-join place.

Similarly, from $C$ there exist two rules $\left(r_{3}\right.$ and $\left.r_{6}\right)$, leading to $D$ and $E$ respectively. Together with the common end transition $t_{e}$ and place $p_{e}$, the resulting Petri net would look as follows:

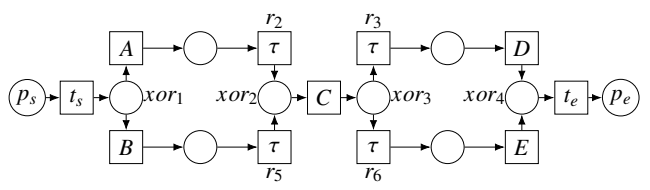

Fig. 4: Merged Petri net $N_{M}$ without preserving dependencies.

However, we want to obtain a condition-free representation of subsequent exclusive branches that maintain their dependencies without duplicating activities. Although task $C$ should not be duplicated in the merged Petri net, dependencies between $A$ and $D$ should remain intact (similar for $B$ and $E$ ). In the example shown in Figure $4, A$ could be followed by $E$, which is incorrect as both correspond to different cases.

When the presets and/or postsets of common transitions (i.e. $t_{s}, C$ and $t_{e}$ ) are different, additional $\tau$ transitions are required to enforce these case-specific dependencies. For instance, transition $t_{s}$ has different postsets for $c_{1}$ and $c_{2}$.

As such, we add $C_{1}^{s}$ and $C_{2}^{s}$ after $x_{0}$, but before $A$ and $B$ respectively. Subsequently, we can add two $\tau$ transitions representing $c_{1}$ and $c_{2}$. These two transitions are mutually exclusive (as shown in Figure 5), such that the chosen path after

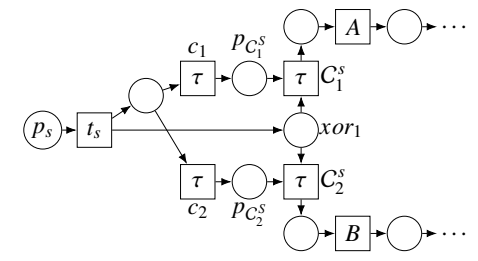

Fig. 5: Enforcing cases (splits). 
$x_{1} r_{1}$ can be determined by the case currently being executed. That is, $C_{1}^{s}$ may only fire with a token in $x_{0} r_{1}$ and after $c_{1}$ has fired. This way, the execution of $A$ is specific to case $c_{1}$, whereas the execution of $B$ is specific to case $c_{2}$.

In case of common transitions with different presets, a similar procedure is required to ensure that all incoming conditions are satisfied. For example, we may have a similar rule in two cases, where in one case a certain input condition is true at the initial state and, in the other case, that condition may be derived by another rule. Consequently, the inputs of that common transition may differ, requiring a synchronisation step to only proceed when all incoming transitions have fired. As such, we add a $\tau$ transition $C_{i}^{j}$ for each incoming path.

The full resulting Petri net $N_{M}$ is represented in Figure 7. Note that the purpose of the $C^{s}$ transitions here is immediately clear, as the co-occurrence of $A$ and $D$ for case $c_{1}$ (and $B$ and $E$ for case $c_{2}$ ) is warranted. In Figure 7 , the ex-

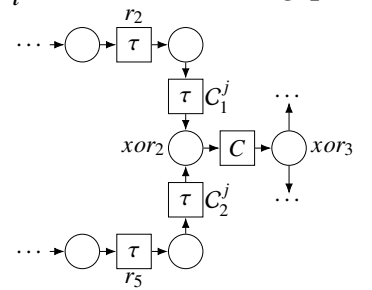
ecution of the tasks in case $c_{1}$ is highlighted in purple, while the auxiliary routing to preserve dependencies is highlighted in orange.

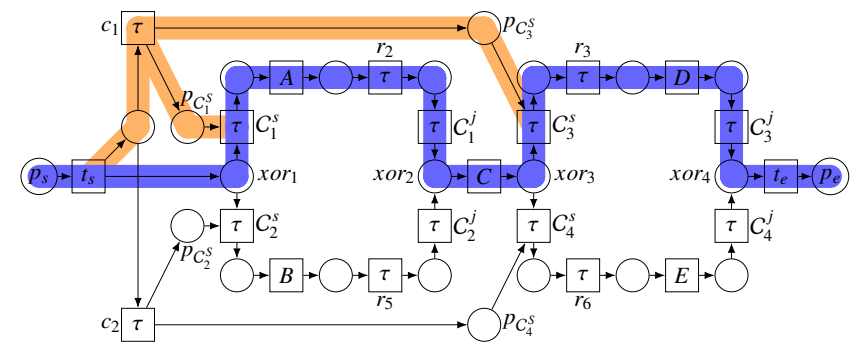

Fig. 7: Merged Petri net $N_{M}$ from $N_{1}$ and $N_{2}$.

Finally, after all Petri nets $N_{i}$ are merged, we optimise the merged Petri net $N_{M}$ by removing all $\tau$ transitions with $|\bullet \tau|=|\tau \bullet|=1$ from $N_{M}$ (i.e. we remove $C_{1}^{j}, C_{2}^{j}, C_{3}^{j}$ and $C_{4}^{j}$ in Figure 7). The resulting optimised merged Petri net can be represented graphically as shown in Figure 8.

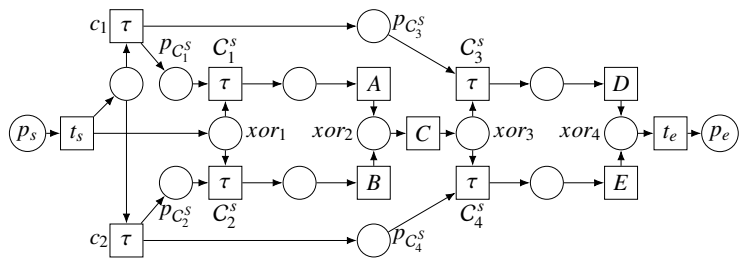

Fig. 8: Optimised Petri net $N_{M}$.

\section{Related WORK}

Automated planning is a technique to organise actions with the aim of achieving some pre-specified goal starting from the current state of the system [11]. Each action features a set of preconditions that have to be satisfied prior to its execution and a set of effects, which specify the state change resulting from its execution. There are frameworks to generate plans (e.g., KPG [14] and Golog [15]), but these are typically based on classical AI planning and do not consider norms. In addition, many automated planning approaches in the business

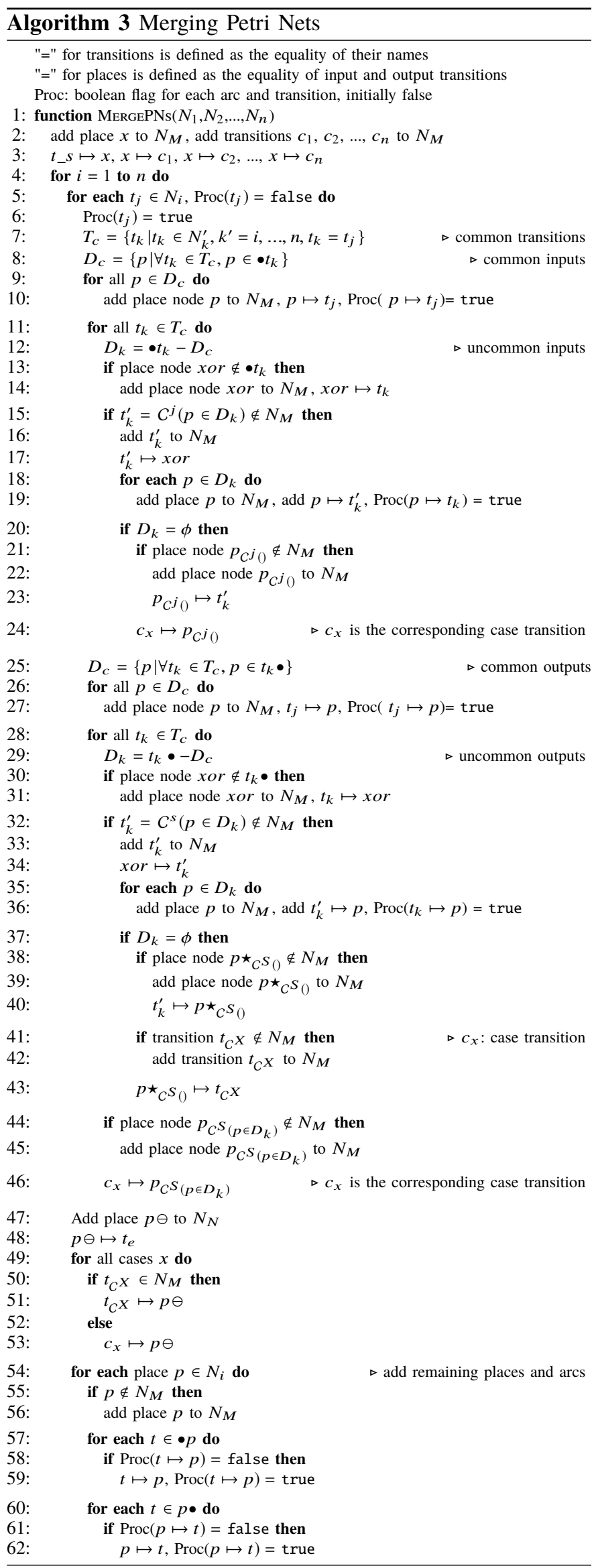


process domain focus on runtime adaptation of pre-specified processes, concerning runtime repair instead of design time process generation based on rules (see e.g. [3][4]). Automated planning techniques require a goal to be specified along with an initial state. However, in case of multiple initial states, multiple possible plans have to be generated (i.e. all possible traces for each existing input case), that need to be merged in order to represent a full business process [5]. As such, providing a business process model that supports all possible traces as specified by the rules remains a challenge.

DECLARE provides an approach for declarative specifications of business processes by means of constraints [16] and graphical representations are available to visualise the constraints and the activities in the model [17]. However, the graphical representation shows the exact constraints and does not provide an actual process model imposed by the rules (i.e. a model showing the possible traces). In [6], an approach is presented to convert a DECLARE model to a behaviourally equivalent Petri net. However, this approach leads to multiple transitions representing the same activity and, therefore, a highly complicated model. Our approach, on the other hand, provides a model without additional duplication of transitions. In [18], an extension is developed on top of BPMN, BPMN$\mathrm{D}$, which supports declarative process modelling. It allows to transform DECLARE models into readable BPMN-D models. This approach, however, does not support concurrency. In addition, DECLARE is based on Linear Temporal Logic which is unable to represent norms with a certain form even for trivial processes [19] and can, as such, not be used for this purpose.

Sardina et al. [20] provide an account of goals in the view of declarative aspects by integrating BDI failure mechanisms with Hierarchical Task Network (HTN) planning techniques. HTN planning is notoriously undecidable even if no variables are allowed, or PSPACE-hard if restrictions are given either on non-primitive tasks or on the ordering of tasks. The main feature of their $\operatorname{CAN}^{\mathcal{A}}$ is its detailed operational semantics where, if a plan fails, alternative plans for achieving the goal are tried. Compared to theirs, the approach presented in this paper has the advantage that we generate all possible plans at design time.

Finally, in [21][22], a declarative approach is used to create compliant business processes on the fly using BPMN notation. Although their approach has been proven sound, it lacks a formal proof of completeness. Moreover, our choice of using Petri nets instead of BPMN notation facilitates us to prove the formal verification of soundness and completeness.

\section{Conclusion}

Enterprises spend a significant effort to maintain their business processes to be efficient and compliant with the norms regulating their operational environment. Declarative formalisms are able to describe both the means an organisation uses to reach its business objectives, and the norms impacting the organisational environment. Although extremely important, it is difficult to adhere to such rules, as the amount rules is usually very large. In addition, understandability of such large sets of rules is generally very low.

This paper resolves these problems by presenting a new method to automatically and efficiently transform a set of business rules into a business process (in the form of a Petri net). The approach presented is particularly useful for organisations as it ensures that their business processes are compliant by design with the normative system and resilient to changes in regulations or corporate policies. More specifically, rule changes can be automatically adopted in the design of the affected business processes and their affect on the actual process flow can be immediately visualised.

In this paper, loops are not considered in the rules. Although the rules themselves allow to specify loops, the use of derivations prevented us from including such behaviour in the final model. Furthermore, the coverage of the applicability of the resulting Petri net is limited to the cases explicitly given by the designers. However, the constructions could be used at runtime to generate a Petri net corresponding to a set of rules and an input case on the fly. Additionally, future lines of research will lead us to an elaborate empirical evaluation to study the understandability and usability of the resulting Petri net compared to the rule set as perceived by end users.

\section{REFERENCES}

[1] T. Morgan, Business rules and information systems: aligning IT with business goals. Addison-Wesley Professional, 2002.

[2] G. Governatori and S. Sadiq, "The journey to business process compliance," in Handbook of Research on BPM (J. Cardoso and W. van der Aalst, eds.), ch. 20, pp. 426-454, IGI Global, 2009.

[3] N. R. T. P. van Beest, E. Kaldeli, P. Bulanov, J. C. Wortmann, and A. Lazovik, "Automated runtime repair of business processes," Information Systems, vol. 39, pp. 45-79, 2014.

[4] A. Marrella and M. Mecella, "Continuous planning for solving business process adaptivity," in BPMDS-11, 2011.

[5] B. Heinrich and D. Schön, "Automated planning of process models: The construction of simple merges," in ECIS 2016, 2016.

[6] J. Prescher, C. Di Ciccio, and J. Mendling, "From declarative processes to imperative models.," SIMPDA, vol. 14, pp. 162-173, 2014.

[7] W. M. P. van der Aalst, "The application of petri nets to workflow management," Journal of Circuits, Systems, and Computers, vol. 8, no. 1, pp. 21-66, 1998.

[8] M. Hashmi, G. Governatori, and M. T. Wynn, "Normative requirements for regulatory compliance: An abstract formal framework," Information Systems Frontiers, vol. 18, no. 3, pp. 429-455, 2016.

[9] G. Governatori, F. Olivieri, A. Rotolo, S. Scannapieco, and M. Cristani, "Picking up the best goal - an analytical study in defeasible logic," in RuleML 2013, pp. 99-113, Springer, 2013.

[10] G. Governatori, F. Olivieri, S. Scannapieco, A. Rotolo, and M. Cristani, "The rationale behind the concept of goal," TPLP, vol. 16, no. 3, pp. 296-324, 2016.

[11] M. Ghallab, D. Nau, and P. Traverso, Automated Planning: Theory and Practice. Morgan Kaufmann, 2004.

[12] C. A. Petri, Communication with automata. $\mathrm{PhD}$ thesis, Universität Hamburg, 1966.

[13] R. Dijkman, M. Dumas, and C. Ouyang, "Semantics and analysis of business process models in bpmn," Information and Software Technology, vol. 50, no. 12, pp. 1281-1294, 2008.

[14] A. C. Kakas, P. Mancarella, F. Sadri, K. Stathis, and F. Toni, "The KGP model of agency," in ECAI, pp. 33-37, IOS Press, 2004.

[15] A. Gabaldon, "Making golog norm compliant," in CLIMA, vol. 6814 of Lecture Notes in Computer Science, pp. 275-292, Springer, 2011.

[16] M. Pesic, H. Schonenberg, and W. M. Van der Aalst, "Declare: Full support for loosely-structured processes," in EDOC 2007, pp. 287-287, IEEE, 2007.

[17] M. Westergaard and F. M. Maggi, "Declare: A tool suite for declarative workflow modeling and enactment.," BPM Demos, vol. 820, pp. 1-5, 2011.

[18] G. De Giacomo, M. Dumas, F. M. Maggi, and M. Montali, "Declarative process modeling in BPMN," in CAISE, pp. 84-100, Springer, 2015.

[19] G. Governatori and M. Hashmi, "No time for compliance," in EDOC 2015, pp. 9-18, IEEE, 2015.

[20] S. Sardiña and L. Padgham, "A BDI agent programming language with failure handling, declarative goals, and planning," JAAMAS, vol. 23, no. 1 , pp. 18-70, 2011.

[21] F. Olivieri, G. Governatori, S. Scannapieco, and M. Cristani, "Compliant business process design by declarative specifications," in PRIMA 2013, pp. 213-228, 2013.

[22] F. Olivieri, M. Cristani, and G. Governatori, "Compliant business processes with exclusive choices from agent specification," in PRIMA 2015, pp. 603-612, 2015. 